\title{
Emerging Trends in Epidemiology of Hepatitis B Virus Infection
}

\author{
Cristina Stasi $*^{1,2}$, Caterina Silvestri ${ }^{1}$ and Fabio Voller ${ }^{1}$ \\ ${ }^{1}$ Observatory of Epidemiology, Regional Health Agency of Tuscany, Florence, Italy; ${ }^{2}$ Department of Experimental and \\ Clinical Medicine, University of Florence, Florence, Italy
}

\begin{abstract}
Although a vaccine against hepatitis B virus (HBV) has been available since 1982, the prevalence of adults with chronic HBV infection in sub-Saharan Africa and East Asia is still estimated at $5-10 \%$. A high rate of chronic infections is also found in the Amazon and the southern parts of eastern and central Europe. In the Middle East and the Indian subcontinent, the prevalence is $2-5 \%$. Less than $1 \%$ of the population of Western Europe and North America is chronically infected. Given the high prevalence of infections (such as hepatitis) among inmates, prison is considered a reservoir for facilitating such infections. Based on these premises, this current review examines and discusses emerging trends in the epidemiology of HBV infection, with particular attention to HBV infection in prison. The hepatitis B surface antigen (HBsAg) prevalence in prisoners in west and central Africa is very high $(23.5 \%)$. The Centers for Disease Control and Prevention has highlighted the importance of HBV blood screening and subsequent anti-HBV vaccination in the prison population. The vaccination was recommended for all inmates, representing an opportunity to prevent $\mathrm{HBV}$ infection in a high-risk population. In these subjects, an accelerated hepatitis B immunisation schedule may result in rapid seroconversion for early short-term protection. Therefore, it is necessary to seek collaboration among public health officials, clinicians and correctional authorities to implement a vaccination programme.
\end{abstract}

Citation of this article: Stasi C, Silvestri C, Voller F. Emerging trends in epidemiology of hepatitis B virus infection. J Clin Transl Hepatol 2017;5(3):272-276. doi: 10.14218/ JCTH.2017.00010.

\section{The state of the art}

The vaccine against the hepatitis B virus (HBV) has been available since 1982 . It is $95 \%$ effective in preventing infection as well as the development of chronic disease and

Keywords: Chronic hepatitis B virus infection; Public health; Anti-HBV vaccine; Epidemiology; Prison.

Abbreviations: anti- $\mathrm{HBc}$, hepatitis $\mathrm{B}$ core antigen; APRI, aspartate aminotransferase platelet ratio index; $C D C$, Centers for Disease Control and Prevention; HbeAg, hepatitis B e antigen; HbsAg, hepatitis B surface antigen; HBV, hepatitis B virus; $\mathrm{HCV}$, hepatitis C virus; HIV, human immunodeficiency virus; OBIs, 'occult' HBV infections; MSM, men who have sex with men; WHO, World Health Organization. Received: 14 February 2017; Revised: 15 April 2017; Accepted: 21 April 2017

*Correspondence to: Cristina Stasi, Observatory of Epidemiology, Regional Health Agency of Tuscany, 50141 Florence, Italy. Tel: +39-55-4624385, Fax: +39-55-4624330, E-mail: cristina.stasi@gmail.com liver cancer due to $\mathrm{HBV}^{1}$ The vaccine has reduced HBV's prevalence and socioeconomic impact in industrialised countries, and systematic reviews of serological biomarkers suggest that about 257 million people are currently living with HBV. ${ }^{1}$

$\mathrm{HBV}$ infection is widespread, with a prevalence $>8 \%$ in parts of sub-Saharan Africa such as West Africa-particularly, Burkina Faso, Ivory Coast, Gambia, Ghana, Guinea, Liberia, Mali, Mauritania, Niger, Nigeria, Senegal, Sierra Leone, TogoSouthern Sudan, Angola, Uganda and Somalia. An intermediate prevalence $(2-7.99 \%)$ is present in some regions of the eastern Mediterranean (e.g. Tunisia), Central Asia (e.g. Kazakhstan), Southeast Asia (e.g. Thailand, Bhutan and Bangladesh), China, parts of South America (e.g. Colombia) and in some European countries (e.g. Albania, Bulgaria, Romania and Turkey). A low prevalence is present $(<2 \%)$ in some parts of North America (e.g. the United States, Canada and Mexico), in some European countries (e.g. Belgium, Czech Republic, Denmark, France) and in Australia. ${ }^{2}$

In the World Health Organization (WHO) European Region, approximately 13 million people are chronically infected with HBV, which leads to about 60,000 deaths a year from hepatitis B-related liver cancer and cirrhosis. ${ }^{3}$ Inmates in correctional facilities bear a greater burden of chronic viral infections and sexually transmitted diseases. ${ }^{4,5}$ In general, high levels of viremia or infection, contracted at a young age, affect mostly males and are associated with an increased risk of death or developing hepatocellular carcinoma. ${ }^{6}$ The impact of screening programmes for viral hepatitis and HBV vaccination has significantly reduced viral hepatitis.

Universal HBV vaccination programmes for infants, with the first dose given at birth, have been highly effective in reducing the prevalence of HBV in many countries. By 2015, 47 of the 53 countries in the WHO European Region had conducted universal HBV vaccination; however, only 26 were vaccinating new-borns, while the other 21 started vaccination at age 2 months of age or later. ${ }^{3}$ Vaccination of adults who are at high risk for HBV infection can prevent transmission of HBV. People at high risk for $\mathrm{HBV}^{1}$ including people interned in prison, are listed in Table 1. Fig. 1 shows a proposed algorithm after screening for HBV infection in prison.

In March 2015, the WHO published the first guidelines for the prevention, care and treatment of people living with chronic HBV infection, ${ }^{7}$ complementing the published 2014 guidance by the WHO on prevention, care and treatment of infection due to the hepatitis $\mathrm{C}$ virus ( $\mathrm{HCV}$ ). The primary goal of these guidelines is to provide clear evidence about the choice of drugs to be used and the criteria for identifying patients for the treatment, particularly in low- and middle-income 
Stasi C. et al: Emerging trends for HBV infection

Table 1. People at high risk for HBV ${ }^{1}$

People who frequently require blood or blood products
Dialysis patients
Recipients of solid organ transplantations
People interned in prisons
People who inject drugs
People with household and sexual contact of people with
chronic HBV infection
People with multiple sexual partners
Health care workers
Travellers who have not completed their vaccination

countries where resources are limited. The guidelines also consider the needs of specific populations, such as co-infected people with human immunodeficiency virus (HIV), children, adolescents and pregnant women. These guidelines are based on a public health approach to the use of antiviral drugs for treatment of chronic HBV infection, taking into consideration the feasibility and effectiveness of the treatment even in countries with limited resources-for example, in areas with limited access to diagnostic methods, such as HBV DNA detection or liver biopsy. ${ }^{7}$

Based on these presuppositions, this review examines and discusses emerging trends in epidemiology of HBV infection, with a particular focus on infection and vaccination against $\mathrm{HBV}$ in people at high risk of infection, such as inmates.

\section{HBV transmission}

The virus owes its remarkable diffusion to its environmental resistance (about 7 days at room temperature). However, after introduction of the vaccination, significant reduction in its transmission has been detected. The detection of HBV in blood donors is achieved by screening for hepatitis B surface antigen (HBsAg) and for antibodies against hepatitis $B$ core antigen (anti-HBc). However, the availability of HBV nucleic acid testing for the screening of blood donors has led to a further reduction in HBV prevalence.

Use of genomic detection was added to HBsAg screening to identify infections by detecting window-period infections and 'occult' HBV infections (OBIs), characterised by undetectable HBsAg and presence of HBV DNA in the liver (with detectable or undetectable HBV DNA in the serum). When detectable, the amount of HBV DNA in the serum is usually very low $(<200 \mathrm{IU} / \mathrm{mL})$. On the basis of the HBV antibody profile-that is, anti-HBc and/or anti-HBs positive; anti$\mathrm{HBC}$ and anti-HBs negative-OBI may be distinguished as seropositive-OBI or seronegative-OBI, respectively. ${ }^{8}$

Although the prevalence of OBI has been described in different areas of the world and in varied categories, the differences of both laboratory techniques used and subject selection criteria have not allowed for meaningful comparisons. However, OBI prevalence seems to be higher in subjects at high risk for HBV infection and with liver disease compared to subjects at low risk of infection and without liver disease. ${ }^{8}$

In Italy, the prevalence of confirmed HBV-positive infection in 2013 appeared to be $154.6 / 100,000$ in first-time donors and incidence was $10.2 / 100,000$ in repeat tested donors. ${ }^{9}$ Ural and Findik ${ }^{10}$ have studied 48 subjects with persistent isolated core antibody (anti-HBc). All were vaccinated with recombinant hepatitis $B$ vaccine at 0,1 and 2 months. Anamnestic response in the isolated anti-HBc group was $41.6 \%$, and the primary response was $47.9 \%$. No vaccine response was found in $10.4 \%$.

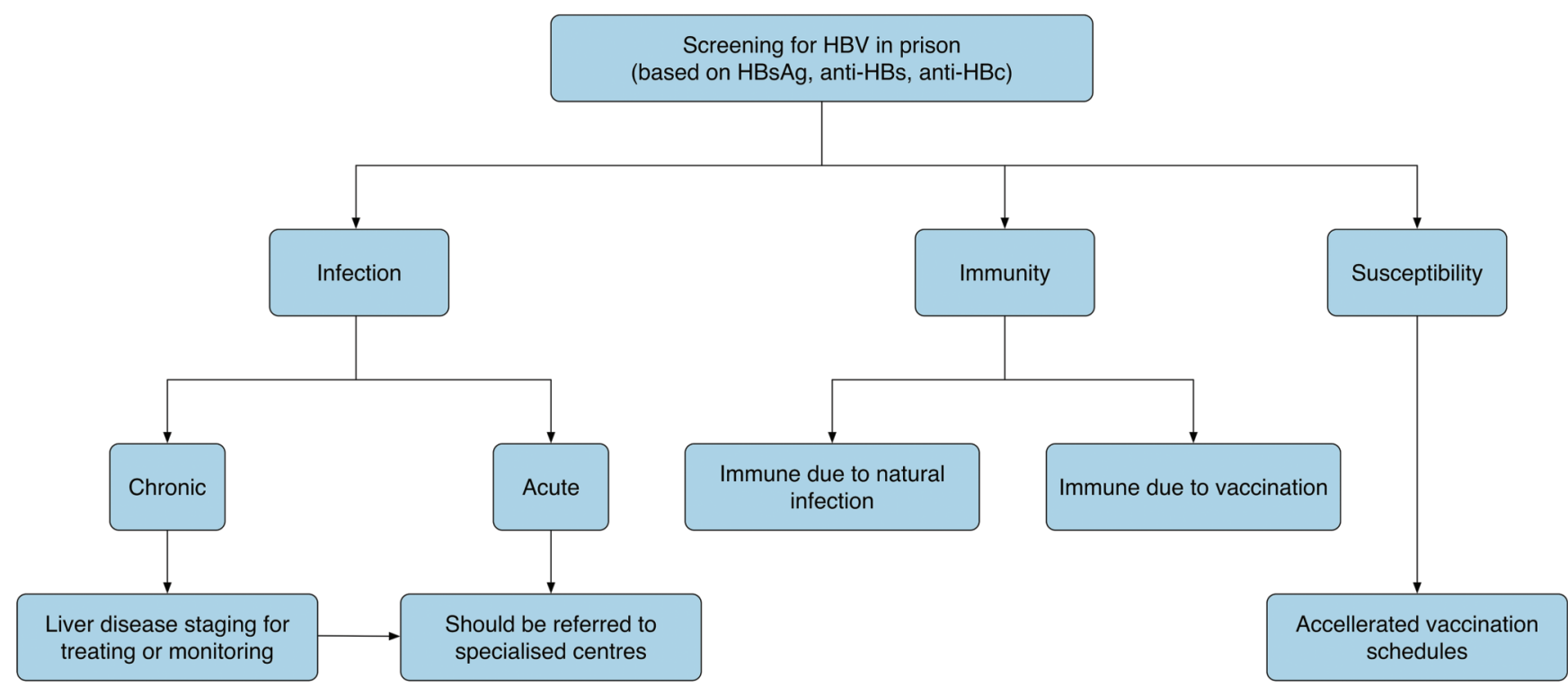

Fig. 1. Proposed algorithm after screening for $\mathbf{H B V}$ infection in prisoners. A subject with anti-HBs and IgM anti-HBC negativity, but $H B s A g$ and anti-HBC positivity is chronically infected. A subject with anti-HBs negativity, and HBsAg and IgM anti-HBc positivity is acutely infected. A subject with HBsAg negativity, and anti-HBc and anti-HBs positivity is immune due to natural infection. A subject with only anti-HBs positivity is immune due to $\mathrm{HB}$ vaccination. A subject with all markers showing negativity is susceptible to infection. Anti-HBc positivity, in the presence of HBsAg and anti-HBs negativity, can be found in the following cases: 1 ) resolved infection; 2) false positivity of anti-HBc; 3) resolving acute infection; and 4) occult infection. It could be useful to evaluate other markers (HBeAg, anti-HBe) and determine the viremia (HBV-DNA) and anti-HCV. 
Fig. 2 shows the possible response to vaccination in anti-HBc positive subjects. Universal infection control practices (i.e. the use of separate rooms and separate machines for HBsAg-positive patients) have dramatically reduced the incidence of HBV in dialysis patients; although, outbreaks continue to occur in the centres of chronic dialysis. Since 1980, the anti-HBV vaccination was recommended for all subjects in serum-negative dialysis and staff. However, in long-term dialysis, patients have a poor immune response to the HBV vaccine in comparison to healthy subjects; in fact, the rate of immunisation and the anti-HBs titre is lower after completion of the vaccination programme. ${ }^{11}$

Practitioners have used various approaches to improve the immunogenicity, such as boosters based on antibody titre, recombinant vaccines and use of immunomodulators in conjunction with vaccine, to increase the efficacy in immunocompromised patients. HBV transmission by haemodialysis is more evident in developing countries. The review of Fabrizi et al. ${ }^{11}$ shows HBV prevalence of between $1.7 \%$ and $15.4 \%$ in Asia and in developing countries.

The results of the Dialysis Outcomes and Practice Patterns Study conducted at 308 dialysis centres in the industrialised world (France, Germany, Italy, Spain, United Kingdom, Japan and the United States) on a total of 8,615 haemodialysis patients showed an average prevalence of $3 \%$, with a median of $1.9 \% .^{12} \mathrm{Di}$ Napoli et al. ${ }^{13}$ analysed data from the Lazio (region of Italy) Dialysis and Transplantation Registry from 2004 to 2012; in 2012, there were 4,072 patients on chronic dialysis. Foreign patients compared to Italian patients were younger and had a higher prevalence of HBV $(53,816.3$

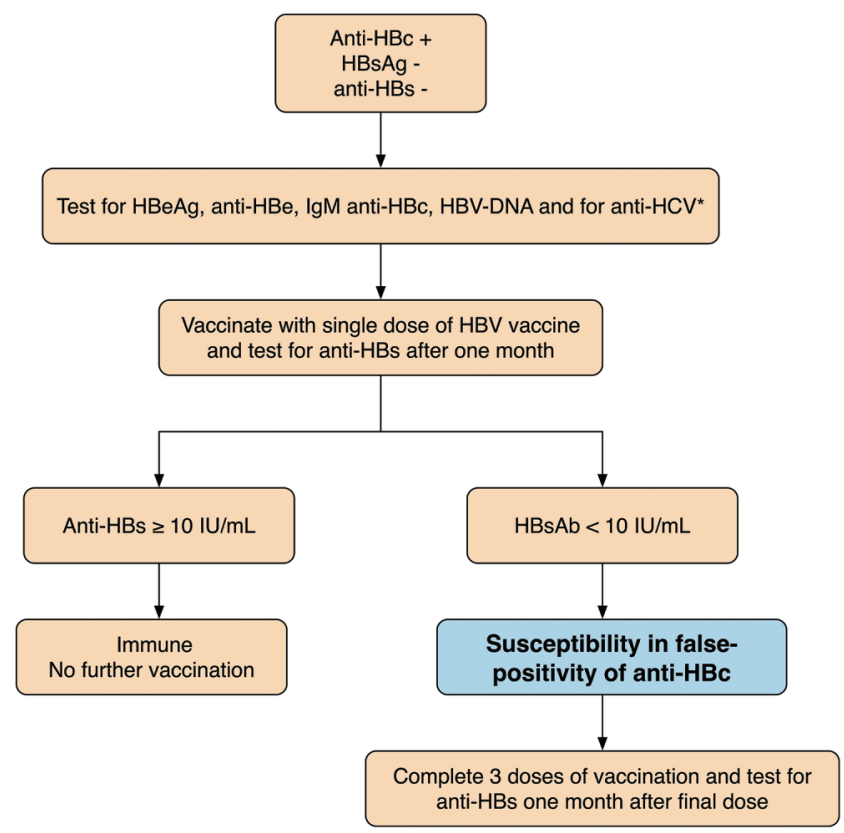

Fig. 2. Response to vaccination in anti-HBc positive subjects. In cases of isolated anti-HBc, test for $\mathrm{HBeAg}$, anti-HBe and HBV-DNA to exclude HBV infection test for anti-HCV as well. IgM anti-HBc positivity ( $\leq 6$ months) indicates acute HBV infection. In cases of isolated presence of anti-HBC (IgM anti-HBC-), vaccination with a single dose of HBV vaccine could be indicated. Anti-HBs $\geq 10 \mathrm{IU} / \mathrm{mL}$ is due to the anamnestic response and this titre indicates that subjects are immune. Anti-HBs $<10 \mathrm{IU} / \mathrm{mL}$ could indicate susceptibility to infection; In subjects who develop a primary response to 3 doses of vaccination, an isolated anti-HBc positive is probably false-positive. No response to vaccination suggests occult HBV infection. * due to the frequency of HBV-DNA in liver biopsy samples from HCV patients. ${ }^{28}$ patients vs. $68,713.6$ patients), involved more frequently women than men $(42.7 \%$ vs. $37.7 \%)$, showed HBsAg positivity $(18.1 \%$ vs. $13.9 \%)$ and were non-vaccinated $(26.8 \%$ vs. $20.9 \%$ ).

Worldwide in 2010, 1.2 million (range, 0.3-2.7 million) of injection drug users were $\mathrm{HBsAg-positive,} \mathrm{with} \mathrm{a} \mathrm{weighted}$ overall prevalence of $8.4 \%$. The $\mathrm{HBsAg}$ prevalence was measured in 59 countries (harbouring $73 \%$ of the world's population), midpoint prevalence estimates of $5-10 \%$ in 21 countries and more than $10 \%$ in 10 countries. ${ }^{14}$

The risk of mother-to-child transmission is related to the state of HBV replication of the mother that correlates with the presence of hepatitis B e antigen (HBeAg). In fact, $90 \%$ of $\mathrm{HBeAg}$-positive mothers transmit HBV infection to their newborns compared to $10 \%-20 \%$ of HBeAg-negative mothers. ${ }^{15}$ $\mathrm{HBsAg}, \mathrm{HBeAg}$ and HBV DNA can be detected in colostrum, and higher titres of $\mathrm{HBsAg}$ and $\mathrm{HBeAg}$ are found in mothers with high levels of serum HBV DNA, suggesting that breast milk could be an important vehicle for HBV transmission. ${ }^{16}$ Some studies $^{16}$ have shown that women with high levels of HBV DNA have an increased risk of transmitting the infection to the child. Therefore, antiviral treatment of the HBV-infected mother with nucleos(t)ide analogues only when indicated and passive and active immunoprophylaxis to new-borns is an excellent way to block natal and perinatal transmission. ${ }^{16}$

Heterosexual-and especially homosexual-activity is an important route of transmission. The HBsAg prevalence estimates range from $<1 \%$ to $4 \%$ in men who have sex with men (MSM) in 3 of the 34 countries included in the review by Hahné et al. ${ }^{17}$ The HBsAg prevalence in MSM was estimated to be 22 times higher than the general population of countries that had available data. ${ }^{17}$ Therefore, the routes of transmission outline the changes in HBV epidemiology.

Prisoners are at high risk of HBV infection due to social and environmental risk factors (i.e. use of intravenous drugs, unprotected sex, tattooing using non-sterilised needles), in addition to specific features of the prison environment (i.e. restricted space and overcrowding). In prison, prevention programmes for vaccination against HBV could be both a challenge and an opportunity; many people who do not otherwise have access to health care can be reached and followed for a certain period, although the environment itself presents an increased risk for HBV transmission. ${ }^{5,18}$

\section{Screening and diagnosis}

In a systematic review, Geue et al. ${ }^{19}$ evaluated 15 studies on HBV screening, out of 2,284 initially considered. The authors stressed the differences among the various population groups surveyed; in particular, some populations studied in the past (i.e. the general population) should not be screened in the future because the screening is not cost-effective. New evidence suggests that screening activities conducted in migrant populations might be a better cost-effective strategy. This result, however, does not show changes in the use of different economic models adopted, evaluation of the quality adjusted life years, the life-years gained, the number of cases detected, or the number of infections avoided.

The WHO guidelines ${ }^{7}$ promote the use of simple, noninvasive diagnostic tests to assess the stage of liver disease, identify patients who need treatment and prioritise those with more advanced liver disease and a higher risk of mortality.

The aspartate aminotransferase platelet ratio index (APRI) is recommended as a non-invasive diagnostic test for the 
presence of cirrhosis (APRI score $>2$ ), which can assess the stage of disease in adult patients in countries with limited resources. The non-invasive methods (e.g. Fibroscan or FibroTest) can be chosen in those countries where these tests are available and the cost does not represent a major restriction (conditional recommendation, low quality of evidence).

The Centers for Disease Control and Prevention (CDC) has highlighted the importance of HBV blood screening and subsequent anti-HBV vaccination in the prison population for all juveniles and adults who have received a medical evaluation, unless they have proof of completion of the vaccine series or serologic evidence of immunity to infection. ${ }^{20}$ According to the $\mathrm{CDC}^{20}$ and Almasio et al., ${ }^{21} \mathrm{HBV}$ screening is recommended for all inmates due to the numerous risk factors to which they are exposed. The findings outlined the importance of screening for HBsAg and anti-HBs to differentiate between infection and immunity. Fig. 1 shows a proposed algorithm after screening for HBV infection in prison.

\section{HBV infection's emerging trends in prison}

The HBsAg prevalence in prisoners in west and central Africa is very high $(23.5 \%)$. High levels of chronic HBV infection have also been reported in east and southern Africa (5.7\%) and in Eastern Europe and central Asia (10.4\%). ${ }^{22}$ The vaccine against $\mathrm{HBV}$ protects against infection and against liver damage caused by the virus. Almasio et al. ${ }^{21}$ recommended vaccination against $\mathrm{HBV}$ in immigrants who belong to the following categories: originated from highly endemic areas; drug addicts; non-immunised prisoners; partners of an infected person; or patients with chronic liver disease unrelated to HBV. For all these groups, a short cycle of vaccinations should be offered to allow for a sufficiently high compliance rate and to avoid losses. ${ }^{21,23}$

Van Herck et al. ${ }^{24}$ showed that an accelerated schedule $(0,1,2$ and 12 months) or super-accelerated schedule $(0,7,21$ days and after 12 months) can result in higher proportions of healthy vaccinated reaching anti-HBs antibody levels $\geq 10 \mathrm{IU} / \mathrm{L}$ more rapidly. For rapid seroconversion and almost immediate protection in the short term, the more accelerated schedule should be used for at-risk groups. Since long-term protection data for these more accelerated schedules have not been reported, a fourth dose at month 12 is still required.

In sum, vaccination should be recommended to all inmates, which represents an opportunity to prevent HBV infection in persons at high risk. In these subjects, an accelerated hepatitis $B$ immunisation schedule may result in a rapid seroconversion and early short-term protection. Although hepatitis $B$ vaccination of inmates has been recommended since 1982, when the vaccine first became available, only some countries routinely vaccinate inmates. ${ }^{25}$ In Italy, a Ministerial Decree in October 1991 recommended vaccination against HBV in people at risk, including detainees. When a short cycle is indicated, the vaccination currently recommended is that at months 0,1 and 2, and possibly again at month 6 . For those who are homeless, there is an accelerated timetable for immunisation against $\operatorname{HBV}(0,7$ and 21 days) with a booster at 12 months, which translates to higher completion and seroconversion rates than traditional programmes. ${ }^{21}$

A cross-sectional screening study was conducted in Italy through evaluation of blood chemistry and blood-serum markers for HBV infection (in particular for the presence of
HBsAg) in prison. The study identified 4.4\% HBsAg-positive subjects, of whom about $35 \%$ of the foreign imnates. ${ }^{26}$ In 2015, results were published of the multicentre study funded by the Ministry of Health within the 2013 Programme of the Centre for Disease Control, with the diagnoses made according to the International Classification of Diseases (Ninth Revision, Clinical Modification). Data was extrapolated from medical records of 15,751 inmates, and confirmed the high prevalence of chronic HBV infection in $2.0 \%$ of inmates. The study also showed that the prevalence of patients with chronic HBV infection was highly underestimated by the National Health Service, as compared to that which had emerged from seroprevalence studies.

Failure to adhere to the screening programme by inmates, then, is an aim for intervention to reduce the spread of the virus in a community that can become a reservoir of infection. The reasons for nonadherence are found in both personal and institutional aspects, such as the lack of knowledge and awareness (perceived risk) regarding viral hepatitis and the routes of transmission, lack of motivation and/or awareness of the test procedure, concerns about confidentiality, fear of stigma by the prison staff and colleaguesbut also the lack of proactive strategies by the staff and, not least, the lack of continuity of care after release from prison. ${ }^{5,18,27}$ Based on these premises, we are currently conducting within a national project a pilot study (funded by the Ministry of Health) in Tuscany prisons that are applying an accelerated vaccination programme.

\section{Conclusions}

The prison provides an opportunity to promote screening and vaccination in subjects at high risk of contracting HBV to limit the spread of HBV infection. In particular, an accelerated hepatitis $B$ immunisation schedule in these subjects may result in a rapid seroconversion and early short-term protection; although, further longitudinal studies are needed to confirm long-term protection. Therefore, it is necessary to seek collaboration among public health practitioners, clinicians and correctional authorities to implement an effective vaccination programme.

\section{Acknowledgements}

We thank our Honourable Minister of Health for funding this review. For their continued support, we thank Teresa Di Fiandra (General Directorate for Health Prevention, Ministry of Health, Rome, Italy) and the Tuscany Region. This work was supported by the National Centre for Disease Prevention and Control, Ministry of Health, Italy.

\section{Conflict of interest}

The authors have no conflict of interests related to this publication.

\section{Author contributions}

Designed the review, wrote and revised the manuscript (CSt), critically revised the manuscript for important intellectual content (CSi, FV). All authors have given final approval of the version to be published. 


\section{References}

[1] World Health Organization. Hepatitis B. Available from: http://www.who. int/mediacentre/factsheets/fs204/en/, accessed April 2017.

[2] Schweitzer A, Horn J, Mikolajczyk RT, Krause G, Ott J]. Estimations of worldwide prevalence of chronic hepatitis $B$ virus infection: a systematic review of data published between 1965 and 2013. Lancet 2015;386:1546-1555. doi: $10.1016 /$ S0140-6736(15)61412-X.

[3] World Health Organization. Hepatitis B in the WHO European Region. Available from: http://www.euro.who.int/ data/assets/pdf file/0009/ 283356/fact-sheet-en-hep-b.pdf, accessed July 2015.

[4] Voller F, Silvestri C, Martino G, Fanti E, Bazzerla G, Ferrari F, et al. Health conditions of inmates in Italy. BMC Public Health 2016;16:1162. doi: 10. 1186/s12889-016-3830-2.

[5] Stasi C, Silvestri C, Fanti E, Di Fiandra T, Voller F. Prevalence and features of chronic viral hepatitis and HIV coinfection in Italian prisons. Eur J Intern Med 2016;34:e21-e22. doi: 10.1016/j.ejim.2016.04.020.

[6] Taylor BC, Yuan JM, Shamliyan TA, Shaukat A, Kane RL, Wilt TJ. Clinical outcomes in adults with chronic hepatitis $B$ in association with patient and viral characteristics: A systematic review of evidence. Hepatology 2009; 49:S85-S95. doi: 10.1002/hep.22929.

[7] World Health Organization. Guidelines for the prevention, care and treatment of persons with chronic hepatitis B infection. Available from: http://apps. who.int/iris/bitstream/10665/154590/1/9789241549059_eng.pdf, accessed March 2015.

[8] Raimondo G, Allain JP, Brunetto MR, Buendia MA, Chen DS, Colombo M, et al. Statements from the Taormina expert meeting on occult hepatitis $B$ virus infection. J Hepatol 2008;49:652-657. doi: 10.1016/j.jhep.2008.07.014.

[9] Piccinini V, Facco G, Catalano L, Pupella S, Grazzini G. Transfusion transmitted infections in Italy: blood donors epidemiological surveillance. Report 2013. Available from: http://www.iss.it/binary/publ/cont/14_26_web.pdf, accessed December 2014.

[10] Ural O, Findik D. The response of isolated anti-HBc positive subjects to recombinant hepatitis $B$ vaccine. J Infect 2001;43:187-190. doi: 10.1053/ jinf.2001.0878.

[11] Fabrizi F, Martin P, Messa P. Control of HCV, HBV and HIV infections in hemodialysis. G Ital Nefrol 2013;30. pii: gin/30.4.11.

[12] Burdick RA, Bragg-Gresham JL, Woods JD, Hedderwick SA, Kurokawa K, Combe $C$, et al. Patterns of hepatitis B prevalence and seroconversion in hemodialysis units from three continents: the DOPPS. Kidney Int 2003;63: 2222-2229. doi: 10.1046/j.1523-1755.2003.00017.x.

[13] Di Napoli A, Lapucci E, Baglio G, Di Giulio S; Registro Regionale Dialisi e Trapianto del Lazio. Lazio dialysis registry: natives vs foreigners. G Ital Nefrol 2015;32. pii: gin/32.3.8.

[14] Nelson PK, Mathers BM, Cowie B, Hagan H, Des Jarlais D, Horyniak D, et al. Global epidemiology of hepatitis $B$ and hepatitis $C$ in people who inject drugs: results of systematic reviews. Lancet 2011;378:571-583. doi: 10 . 1016/S0140-6736(11)61097-0.

[15] Navabakhsh B, Mehrabi N, Estakhri A, Mohamadnejad M, Poustchi H. Hepatitis $B$ virus infection during pregnancy: transmission and prevention. Middle East J Dig Dis 2011;3:92-102.
[16] Zhang $\mathrm{Z}$, Chen $\mathrm{C}, \mathrm{Li} \mathrm{Z}, \mathrm{Wu} \mathrm{YH}$, Xiao XM. Individualized management of pregnant women with high hepatitis B virus DNA levels. World J Gastroenterol 2014;20:12056-12061. doi: 10.3748/wjg.v20.i34.12056.

[17] Hahné SJ, Veldhuijzen IK, Wiessing L, Lim TA, Salminen M, Laar Mv. Infection with hepatitis $B$ and $C$ virus in Europe: a systematic review of prevalence and cost-effectiveness of screening. BMC Infect Dis 2013;13:181. doi: 10. 1186/1471-2334-13-181.

[18] Sinisgalli E, Silvestri C, Bravi S, Ceccherini V, Tanini T, Bonaccorsi G, et al. Infectious diseases in the Tuscan detention setting: data from the Health Agency of Tuscany. Public Health 2016;141:264-267. doi: 10.1016/j.puhe. 2016.02.019.

[19] Geue C, Wu O, Xin Y, Heggie R, Hutchinson S, Martin NK, et al. Costeffectiveness of HBV and HCV screening strategies-a systematic review of existing modelling techniques. PLoS One 2015;10:e0145022. doi: 10. 1371/journal.pone.0145022.

[20] Weinbaum C, Lyerla R, Margolis HS; Centers for Disease Control and Prevention. Prevention and control of infections with hepatitis viruses in correctional settings. Centers for Disease Control and Prevention. MMWR Recomm Rep 2003;52:1-36; quiz CE1-4.

[21] Almasio PL, Babudieri S, Barbarini G, Brunetto M, Conte D, Dentico P, et al. Recommendations for the prevention, diagnosis, and treatment of chronic hepatitis $B$ and $C$ in special population groups (migrants, intravenous drug users and prison inmates). Dig Liver Dis 2011;43:589-595. doi: 10.1016/j. dld.2010.12.004

[22] Dolan K, Wirtz AL, Moazen B, Ndeffo-Mbah M, Galvani A, Kinner SA, et al. Global burden of HIV, viral hepatitis, and tuberculosis in prisoners and detainees. Lancet 2016;388:1089-1102. doi: 10.1016/S0140-6736(16) 30466-4.

[23] Idilman R, De MN, Colantoni A, Nadir A, Van Thiel DH. The effect of high dose and short interval HBV vaccination in individuals with chronic hepatitis $C$. Am J Gastroenterol 2002;97:435-439. doi: 10.1111/j.1572-0241.2002. 05482.x.

[24] Van Herck K, Leuridan E, Van Damme P. Schedules for hepatitis B vaccination of risk groups: balancing immunogenicity and compliance. Sex Transm Infect 2007;83:426-432. doi: 10.1136/sti.2006.022111.

[25] Centers for Disease Control and Prevention (CDC). Hepatitis B vaccination of inmates in correctional facilities-Texas, 2000-2002. MMWR Morb Mortal Wkly Rep 2004;53:681-683.

[26] Sagnelli E, Starnini G, Sagnelli C, Monarca R, Zumbo G, Pontali E, et al. Blood born viral infections, sexually transmitted diseases and latent tuberculosis in italian prisons: a preliminary report of a large multicenter study. Eur Rev Med Pharmacol Sci 2012;16:2142-2146.

[27] Semaille C, Le Strat Y, Chiron E, Chemlal K, Valantin MA, Serre P, et al. Prevalence of human immunodeficiency virus and hepatitis $C$ virus among French prison inmates in 2010: a challenge for public health policy. Euro Surveill 2013;18. pii: 20524.

[28] Koike K, Kobayashi M, Gondo M, Hayashi I, Osuga T, Takada S. Hepatitis B virus DNA is frequently found in liver biopsy samples from hepatitis $C$ virusinfected chronic hepatitis patients. J Med Virol 1998;54:249-255. 